\title{
Aporte de volumen en el perioperatorio: ¿debemos modificar nuestro enfoque?
}

\author{
MARIO CONCHA P. ${ }^{1}$, VERÓNICA MERTZ K. ${ }^{1}$, LUIS CORTÍNEZ F. ${ }^{1}$, \\ ALVARO ZÚÑIGA D. ${ }^{2}$, GEORGE PINEDO M. ${ }^{2}$
}

Background: During the perioperative period an adequate intravascular volume must be maintained. Current recommendations overestimate perioperative volume requirements. Aim: To compare perioperative volume administration using standard monitoring methods or guided by left ventricular filling parameters. Material and Methods: Twenty-four patients subjected to colon resection were randomized to monitoring by electrocardiography, blood and central venous pressure, or by transesophageal echocardiography. In the latter, volume administration was adjusted to maintain basal values of left ventricular end diastolic volume and cardiac index. Results: Patients with the standard monitoring system and transesophageal echocardiographic monitoring received $21.1 \pm 12$ and $6.3 \pm 2 \mathrm{ml} / \mathrm{kg} / \mathrm{h}$ of fluids during the perioperative period, respectively $(p<0.01)$. Conclusions: The use of transesophageal echocardiography significantly reduced the perioperative fluid administration.

(Rev Med Chile 2011; 139: 1157-1162).

Key words: Central venous pressure; Echocardiography, transesophageal; Intravascular procedures.

\footnotetext{
L
}

a mantención de un adecuado volumen del espacio intravascular constituye un punto pacientes sometidos a gran cirugía. Tanto déficit como excesos en el aporte de volumen han sido relacionados con morbimortalidad ${ }^{1-7}$. Resulta, por lo tanto, de gran importancia disponer de los medios de monitorización que permitan determinar el estado de este compartimento. Desafortunadamente los que utilizamos no son aplicables en clínica o pueden no reflejar la real condición de este $^{8}$. A ello se agrega que las recomendaciones de reposición utilizadas probablemente sobreestiman los requerimientos ${ }^{9,10}$, y que algunas estrategias de reposición se han extendido a situaciones en las que pudieran no ser las más adecuadas ${ }^{11}$. Como resultado de esto, durante el período perioperatorio con frecuencia se administran cantidades de volumen que probablemente son mayores a las requeridas para cumplir los objetivo fisiológicos buscados $^{12,13}$.

El intraoperatorio de la gran cirugía abdominal es considerado un período de elevados requerimientos de reemplazo de volumen intravascular. El poder cuantificar los aportes y pérdidas de volumen, y mantener condiciones hemodinámicas similares entre los grupos comparados, hacen de este período una buena oportunidad de probar la hipótesis que la utilización de medios de monitorización y criterios clínicos habituales, determinan un aumento del volumen administrado, en relación a la cantidad aportada cuando esto se realiza buscando alcanzar un objetivo definido y cuantificable. El objetivo de este estudio fue comparar las diferencias en la cantidad de volumen administrado cuando esto se realiza de acuerdo a métodos y recomendaciones consideradas habituales, o cuando se realiza tratando de mantener 
los valores basales de volumen de llenado ventricular o índice cardiaco (IC).

\section{Pacientes y Método}

El estudio fue aprobado por el Comité de Ética de la Facultad de Medicina de la Universidad Católica de Chile. Los pacientes firmaron consentimiento informado de participación voluntaria. Se estudiaron prospectivamente 24 pacientes programados para cirugía resectiva de colon, clasificados como I o II de la clasificación de la American Society of Anesthesiologists (ASA) ASA I: paciente sano, sólo presenta cuadro quirúrgico que motiva la cirugía, ASA II: paciente con enfermedad sistémica leve, ASA III: enfermedad sistémica moderada, ASA IV: paciente con enfermedad sistémica que pone en riesgo permanente la vida, ASA V: paciente que con o sin cirugía fallecerá en 24 horas. Todos recibieron la misma preparación de colon, que incluía la administración de 1.000 $\mathrm{ml}$ de Ringer lactato durante la noche previa a la cirugía, la que se realizó a primera hora de la mañana. En un grupo la monitorización consistió en electrocardiografía continua, presión arterial no invasiva, presión venosa central (PVC), diuresis, oximetría de pulso, y capnografía (G1). En el otro incluyó además el uso de ecocardiografía transesofágica (ETE) (G2). Los pacientes se asignaron a cada grupo en forma aleatorizada. Todos recibieron la misma técnica anestésica basada en fentanil e isoflurano, cuya administración se ajustó para controlar cambios de presión arterial media
(PAM) y frecuencia cardiaca (FC) mayores de $20 \%$ de los valores basales, definidas estas como el valor promedio de tres determinaciones hechas en reposo el día previo a la cirugía. En G2 se colocó la sonda de ETE una vez terminada la inducción anestésica. Las mediciones basales fueron hechas después de 5 minutos de mantención de PAM y FC dentro de un rango de $\pm 20 \%$ de los valores basales, previo a la incisión quirúrgica, con la mesa en posición neutra, con el paciente en posición quirúrgica con las piernas ligeramente flectadas y separadas (posición de Lloyd-Davies). Se midió el volumen de fin de diástole del ventrículo izquierdo indexado (VFDVII) e índice cardiaco (IC). Todas las determinaciones fueron hechas por el mismo operador (VM). Determinaciones adicionales fueron hechas cada 30 minutos o cada vez que la PAM disminuyó más de $20 \%$ de los valores basales. El VFDVII fue medido en el esófago medio de acuerdo al método de Simpson ${ }^{14}$. El IC fue medido de acuerdo al método descrito por Perrino ${ }^{15}$. En G1 el tipo y cantidad de volumen administrado se realizó de acuerdo a la evaluación del anestesiólogo a cargo, considerando el balance hídrico, y las variaciones de FC, PAM, PVC, y diuresis. Los anestesiólogos a cargo de los pacientes de G1 desconocían el objetivo de este estudio, y sólo se les instruyó para estandarización de la técnica anestésica y de los criterios hemodinámicos para el control de ésta. En G2 se administró una infusión basal de $3 \mathrm{ml} / \mathrm{kg} / \mathrm{h}$ de Ringer lactato. Aportes adicionales se realizaron de acuerdo al algoritmo de la Figura 1. Disminuciones de PAM mayores de

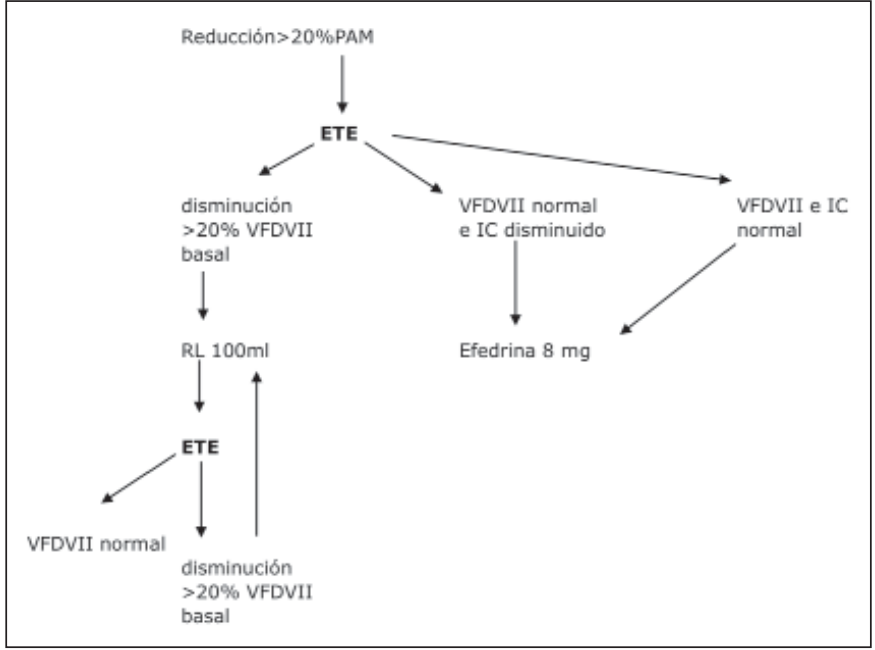

Figura 1. Manejo hemodinámico en pacientes sometidos a cirugía abdominal. PAM: Presión arterial media. VFDVII: Volumen de fin de diástole del ventrículo izquierdo indexado. IC: Índice cardiaco. RL: Ringer lactato. 
$20 \%$ previo a la inserción de sonda de ETE fueron tratadas administrando $100 \mathrm{ml}$ de Ringer lactato y $8 \mathrm{mg}$ de efedrina. Esto fue repetido a los tres minutos en caso de falta de respuesta. Cuando las mediciones realizadas cada 30 minutos mostraban una disminución mayor de 20\% del VFDVII o del IC, se aplicó el mismo algoritmo de la Figura 1. En ambos grupos, la administración de GR se realizó tratando de mantener un hematocrito alrededor de $30 \%$. El tiempo quirúrgico fue definido como el intervalo entre el inicio de la inducción anestésica y el momento de la extubación. No se administraron diuréticos durante el intraoperatorio. Las pérdidas sanguíneas fueron determinadas cada 30 minutos a través de la medición de los frascos de aspiración menos volumen de solución de lavado, y peso de compresas a las que se restó el peso seco. Las complicaciones post operatorias fueron registradas prospectivamente por el equipo quirúrgico, $y$ fueron definidas como cualquier condición clínica que determinara cambios en la terapia o solicitud de exámenes de laboratorio que mostraran hallazgos anormales.

Basados en un estudio previo que mostraba que en cirugía abierta la cantidad de Ringer lactato requerido para mantener un VFDVII o IC similar al basal era de $5,9 \mathrm{ml} / \mathrm{kg} / \mathrm{h}^{16}$, se estimó que se requerían 12 pacientes en cada grupo para detectar una diferencia de al menos $40 \%$ en el total de volumen administrado (potencia $80 \%$, error a 0,05). Los datos están expresados como promedio y desviación estándar. Las comparaciones fueron hechas con la prueba de Wilcoxon sum-rank en muestras independientes o Wilcoxon signed-rank en muestras pareadas. El análisis estadístico fue hecho con el programa $\mathrm{R}$ (acceso libre a través de http://www.r-project.org/). Se consideró significativa una $\mathrm{p} \leq 0,05$.

\section{Resultados}

Se estudiaron 24 pacientes, todos los cuales completaron el período de estudio. Sus datos demográficos se muestran en la Tabla 1. Las cirugías realizadas fueron 8 hemicolectomías y 4 colectomías totales en G1, y en G2 las 12 cirugías fueron hemicolectomías. La cantidad de volumen administrada en cada grupo, pérdidas sanguíneas, requerimientos de efedrina y diuresis se muestran en la Tabla 2. En G1 no hubo diferencias en la cantidad de volumen administrado a los pacientes sometidos a colectomía y hemicolectomía $(19,2 \pm 8,7 \mathrm{ml} / \mathrm{kg} / \mathrm{h}$ y $16,8 \pm 4,4 \mathrm{ml} / \mathrm{kg} / \mathrm{h}$, respectivamente, $p=0,68)$. En $G 1,4$ pacientes recibieron además de Ringer lactato alguna solución coloidal, y un paciente recibió $600 \mathrm{ml}$ de glóbulos rojos.

Tabla 1. Datos demográficos de 24 pacientes sometidos a cirugía de colon

\begin{tabular}{|lcc|}
\hline & G1 & G2 \\
\hline Edad (años) & $60,8 \pm 9,7$ & $59,3 \pm 11,5$ \\
Peso $(\mathrm{kg})$ & $54,3 \pm 9,7$ & $66,7 \pm 12,3^{*}$ \\
\hline Talla $(\mathrm{cm})$ & $162 \pm 5,5$ & $161 \pm 10,2$ \\
Sexo $(\mathrm{m} / \mathrm{f})$ & $7 / 5$ & $8 / 4$ \\
\hline
\end{tabular}

${ }^{*} p<0,05$. Promedio \pm Desviación estándar. m: masculino. f: femenino.

Tabla 2. Volumen administrado, pérdidas sanguíneas, requerimientos de efedrina, y diuresis, en pacientes operados de cirugía de colon

\begin{tabular}{|lcc|}
\hline & G1 & G2 \\
\hline Volumen total administrado $(\mathrm{ml})$ & $2.562 \pm 1.030$ & $1.311 \pm 474^{*}$ \\
\hline Volumen total administrado $\left(\mathrm{ml} \cdot \mathrm{kg}^{-1} \cdot \mathrm{h}^{-1}\right)$ & $21,1 \pm 12$ & $6,3 \pm 2^{*}$ \\
\hline Pérdidas sanguíneas $(\mathrm{ml})$ & $277 \pm 50$ & $302 \pm 126$ \\
\hline Pacientes que requirieron efedrina en la inducción & 8 & 6 \\
\hline Pacientes que requirieron efedrina en la mantención & 6 & 2 \\
Diuresis $\left(\mathrm{ml} \cdot \mathrm{kg}^{-1} \cdot \mathrm{h}^{-1}\right)$ & $1,6 \pm 1,2$ & $0,6 \pm 0,4^{*}$ \\
Duración de la cirugía (horas) & $2,6 \pm 0,9$ & $3,1 \pm 0,7$ \\
\hline
\end{tabular}

$*=p \leq 0,01$, prueba de Wilcoxon para muestras independientes. 
Tabla 3. Mediciones ecocardiográficas en pacientes sometidos a cirugía de colon

\begin{tabular}{|lrr|}
\hline & Basal & \multicolumn{1}{c|}{ Final } \\
\hline VFDVII & $39,2 \pm 5,7$ & $37,5 \pm 1,6$ \\
IC & $3,1 \pm 0,5$ & $3,5 \pm 0,7$ \\
\hline
\end{tabular}

VFDVII $\left(\mathrm{ml} / \mathrm{m}^{2}\right)$ : Volumen de fin de diástole del ventrículo izquierdo indexado. IC ( $\left(1 / \mathrm{m}^{-2}\right)$ : Índice cardiaco.

Todos los pacientes en $\mathrm{G} 2$ requirieron aporte adicional de volumen en al menos una oportunidad. No hubo diferencias en las mediciones ecocardiográficas en los valores basales y finales (Tabla 3). La estadía hospitalaria fue de 9,7 $\pm 3,6$ en G1, y de $11,4 \pm 5,1$ días en $\mathrm{G} 2(\mathrm{p}=0,4)$. Un paciente en cada grupo presentó una infección urinaria, 2 pacientes en G2 presentaron una infección respiratoria, y un paciente en G1 presentó infección de la herida operatoria.

\section{Discusión}

El resultado más importante del estudio fue comprobar que la administración de volumen guiada por un parámetro cuantitativo de las condiciones de llenado ventricular y débito cardiaco, determina una importante disminución del volumen administrado. Resulta importante destacar que la cantidad utilizada en G2 fue significativamente menor a la que ha sido recomendada para este tipo de cirugía, y similar a la utilizada en otros estudios en los cuales se trató de restringir el aporte o se definió un objetivo fisiológico a alcanzar $^{7,16}$. Considerando la evidencia existente en relación a los problemas asociados a un aporte de volumen en exceso ${ }^{1,5,7,17}$, debemos considerar seriamente las limitaciones de los métodos de monitorización que frecuentemente utilizamos, y plantearnos la necesidad de incorporar otros que permitan controlar que una adecuada entrega de oxígeno a los tejidos, objetivo final de la terapia de reemplazo de volumen, se cumpla. La utilización de métodos de monitorización que entreguen esta información, por la complejidad, costo, riesgos e invasividad que representan no se justifican en la mayoría de los pacientes. En otros, sin embargo, la evaluación frecuente de lactato, saturación venosa central, o de las variaciones de la presión de pulso pueden aportar a precisar la estrategia de reposición de volumen.
La disminución del aporte en G2 hace plantear la posibilidad de una disminución del espacio intravascular, que no se evidenció como resultado de activación simpática, la que puede determinar también redistribuciones de flujo que podrían comprometer al territorio esplácnico, renal, piel y músculo, que precozmente son afectados por una disminución del volumen circulante. Aun cuando el diseño del estudio no permite descartar esta posibilidad, esto sería leve ya que disminuciones de hasta $10 \%$ del volumen intravascular no afectan el débito cardiaco o la presión arterial ${ }^{18}$, parámetros ambos que fueron mantenidos cercanos a los basales de acuerdo al protocolo de estudio. En G2 la diuresis fue significativamente menor, y en el límite de lo que habitualmente se acepta. De acuerdo con lo señalado, la mantención de la presión arterial, de llenado ventricular y de IC permiten asumir una adecuada perfusión renal, pudiendo explicarse la disminución de la diuresis por una respuesta fisiológica a la cirugía que promueve la absorción de sodio y agua ${ }^{19}$. Este punto, sin embargo, debe ser específicamente estudiado para el período perioperatorio. Aún cuando ninguno de los pacientes estudiados desarrolló complicaciones relacionadas a un inadecuado reemplazo de volumen intravascular, el número de pacientes estudiados no permite obtener conclusiones.

Una potencial debilidad del estudio es la falta de protocolización en la forma de administrar volumen en G1. Sin embargo, la metodología utilizada trata de representar una manera muy habitual de manejar el aporte de fluidos en el perioperatorio, en la cual criterios ampliamente aceptados pero sin límites precisos en cuanto a lo que se puede aceptar en las variaciones de estos, 0 en las cantidades de fluidos a administrar, terminan provocando acumulación de varios litros de exceso $^{12,13,20,21}$. A la luz de los resultados obtenidos, esto es claramente innecesario para mantener condiciones de llenado e IC normales. La probable sobreestimación de los requerimientos y la escasa capacidad de los medios de monitorización habitual de reflejar las condiciones del compartimento intravascular han sido ampliamente reportadas $^{8,22-27}$ y enfatizan la necesidad de modificar la manera de realizar el aporte de volumen en el perioperatorio. La respuesta acerca de cual es el mejor método aún no está definida, sin embargo, debemos considerar las limitaciones de la monitorización que utilizamos, y los posibles riesgos de 
un aporte excesivo. La utilización de parámetros que nos informen acerca de la efectividad de la entrega de oxígeno, o de la capacidad de respuesta del corazón frente al aporte de volumen ${ }^{28}$ pueden ayudarnos a enfrentar el problema de manera más precisa, evitando el uso de cantidades innecesarias de volumen que podrían estar influyendo en la morbilidad.

\section{Referencias}

1. Brandstrup B, Tonnesen H, Beier-Holgersen R, Hjortso E, Ording H, Lindorff-Larsen $\mathrm{K}$, et al. Effects of intravenous fluid restriction on postoperative complications: comparison of two perioperative fluid regimens: a randomized assessor-blinded multicenter trial. Ann Surg 2003; 238: 641-8.

2. Mythen MG, Webb AR. Perioperative plasma volume expansion reduces the incidence of gut mucosal hypoperfusion during cardiac surgery. Arch Surg 1995; 130: 423-9.

3. Mythen MG, Webb AR. Intra-operative gut mucosal hypoperfusion is associated with increased post-operative complications and cost. Intensive Care Med 1994; 20: 99-104.

4. Sinclair S, James S, Singer M. Intraoperative intravascular volume optimisation and length of hospital stay after repair of proximal femoral fracture: randomised controlled trial. BMJ 1997; 315: 909-12.

5. Lobo DN, Bostock KA, Neal KR, Perkins AC, Rowlands BJ, Allison SP. Effect of salt and water balance on recovery of gastrointestinal function after elective colonic resection: a randomised controlled trial. Lancet 2002; 359: 1812-8.

6. Poelaert JI, Schupfer G. Hemodynamic monitoring utilizing transesophageal echocardiography: the relationships among pressure, flow, and function. Chest 2005; 127: 379-90.

7. Nisanevich V, Felsenstein I, Almogy G, Weissman C, Einav S, Matot I. Effect of intraoperative fluid management on outcome after intraabdominal surgery. Anesthesiology 2005; 103: 25-32.

8. Marik PE, Baram M, Vahid B. Does central venous pressure predict fluid responsiveness? A systematic review of the literature and the tale of seven mares. Chest 2008; 134: 172-8.

9. Hwang G, Marota, JA. Anesthesia for abdominal surgery. In: Hurford WE BM, Davison JK, Haspel KL, Rosow C, ed. Clinical anesthesia procedures of the Massachusetts General Hospital Philadelphia: Lippincott-Raven, 1997: 330-46.
10. Grocott MP, Mythen MG, Gan TJ. Perioperative fluid management and clinical outcomes in adults. Anesth Analg 2005;100:1093-106.

11. Shoemaker WC, Montgomery ES, Kaplan E, Elwyn DH. Physiologic patterns in surviving and nonsurviving shock patients. Use of sequential cardiorespiratory variables in defining criteria for therapeutic goals and early warning of death. Arch Surg 1973; 106: 630-6.

12. Chappell D, Jacob M, Hofmann-Kiefer K, Conzen P, Rehm M. A rational approach to perioperative fluid management. Anesthesiology 2008; 109: 723-40.

13. Rehm M, Haller M, Orth V, Kreimeier U, Jacob M, Dressel $\mathrm{H}$, et al. Changes in blood volume and hematocrit during acute preoperative volume loading with 5\% albumin or $6 \%$ hetastarch solutions in patients before radical hysterectomy. Anesthesiology 2001; 95: 849-56.

14. Lang RM, Bierig M, Devereux RB, Flachskampf FA, Foster E, Pellikka PA, et al Recommendations for chamber quantification. Eur J Echocardiogr 2006; 7: 79-108.

15. Perrino AC, Jr., Harris SN, Luther MA. Intraoperative determination of cardiac output using multiplane transesophageal echocardiography: a comparison to thermodilution. Anesthesiology 1998; 89: 350-7.

16. Concha MR, Mertz VF, Cortínez LI, González KA, Butte JM, López F, et al. The volume of lactated Ringer's solution required to maintain preload and cardiac index during open and laparoscopic surgery. Anesth Analg 2009; 108: 616-22.

17. Lobo DN, Bjarnason K, Field J, Rowlands BJ, Allison SP. Changes in weight, fluid balance and serum albumin in patients referred for nutritional support. Clin Nutr 1999; 18: 197-201.

18. Circulatory Shock and Physiology of its Treatment. In: Guyton AC, ed. Textbook of Medical Phusiology. Tenth ed. Philadelphia: W.B. Saunders Company, 2000: 253-62.

19. Traynor C, Hall GM. Endocrine and metabolic changes during surgery: anaesthetic implications. Br J Anaesth 1981; 53: 153-60.

20. Jacob M, Chappell D, Hofmann-Kiefer K, Conzen P, Peter K, Rehm M. [Determinants of insensible fluid loss. Perspiration, protein shift and endothelial glycocalyx]. Anaesthesist 2007; 56: 747-58, 60-4.

21. Rehm M, Orth V, Kreimeier U, Thiel M, Haller M, Brechtelsbauer H, Finsterer U. Changes in intravascular volume during acute normovolemic hemodilution and intraoperative retransfusion in patients with radical hysterectomy. Anesthesiology 2000; 92: 657-64.

22. Cunnion RE, Natanson C. Echocardiography, pulmonary artery catheterization, and radionuclide cineangiography in septic shock. Intensive Care Med 1994; 20: 535-7. 
23. Stephan F, Flahault A, Dieudonne N, Hollande J, Paillard F, Bonnet F. Clinical evaluation of circulating blood volume in critically ill patients-contribution of a clinical scoring system. Br J Anaesth 2001; 86: 754-62.

24. Hofer CK, Furrer L, Matter-Ensner S, Maloigne M, Klaghofer R, Genoni M, Zollinger A. Volumetric preload measurement by thermodilution: a comparison with transoesophageal echocardiography. Br J Anaesth 2005; 94: 748-55.

25. Spahn DR, Chassot PG. CON: Fluid restriction for cardiac patients during major noncardiac surgery should be replaced by goal-directed intravascular fluid administra- tion. Anesth Analg 2006; 102: 344-6.

26. Gan TJ, Soppitt A, Maroof M, el-Moalem H, Robertson $\mathrm{KM}$, Moretti E, et al. Goal-directed intraoperative fluid administration reduces length of hospital stay after major surgery. Anesthesiology 2002; 97: 820-6.

27. Michard F, Alaya S, Zarka V, Bahloul M, Richard C, Teboul JL. Global end-diastolic volume as an indicator of cardiac preload in patients with septic shock. Chest 2003; 124: 1900-8.

28. Michard F, Teboul JL. Predicting fluid responsiveness in ICU patients: a critical analysis of the evidence. Chest 2002; 121: 2000-8. 